\title{
Uncertainty principles and sum complexes
}

\author{
Roy Meshulam
}

Received: 17 January 2013 / Accepted: 14 February 2014 / Published online: 21 March 2014

(C) Springer Science+Business Media New York 2014

\begin{abstract}
Let $p$ be a prime and let $A$ be a nonempty subset of the cyclic group $C_{p}$. For a field $\mathbb{F}$ and an element $f$ in the group algebra $\mathbb{F}\left[C_{p}\right]$ let $T_{f}$ be the endomorphism of $\mathbb{F}\left[C_{p}\right]$ given by $T_{f}(g)=f g$. The uncertainty number $u_{\mathbb{F}}(A)$ is the minimal rank of $T_{f}$ over all nonzero $f \in \mathbb{F}\left[C_{p}\right]$ such that $\operatorname{supp}(f) \subset A$. The following topological characterization of uncertainty numbers is established. For $1 \leq k \leq p$ define the sum complex $X_{A, k}$ as the $(k-1)$-dimensional complex on the vertex set $C_{p}$ with a full $(k-2)$-skeleton whose $(k-1)$-faces are all $\sigma \subset C_{p}$ such that $|\sigma|=k$ and $\prod_{x \in \sigma} x \in A$. It is shown that if $\mathbb{F}$ is algebraically closed then
\end{abstract}

$$
u_{\mathbb{F}}(A)=p-\max \left\{k: \tilde{H}_{k-1}\left(X_{A, k} ; \mathbb{F}\right) \neq 0\right\}
$$

The main ingredient in the proof is the determination of the homology groups of $X_{A, k}$ with field coefficients. In particular it is shown that if $|A| \leq k$ then $\tilde{H}_{k-1}\left(X_{A, k} ; \mathbb{F}_{p}\right)=0$.

Keywords Uncertainty principle $\cdot$ Simplicial homology

Mathematics Subject Classification $55 \mathrm{U} 10 \cdot 20 \mathrm{C} 05$

\section{Introduction}

Uncertainty type inequalities reflect various quantitative aspects of the general principle that a nonzero function and its Fourier transform cannot both be sharply localized. The first such result is the Fourier theoretic version of the Heisenberg quantummechanical uncertainty principle: If $f \in L^{2}(\mathbb{R})$ satisfies $\|f\|_{2}=1$, then

R. Meshulam $(\bowtie)$

Department of Mathematics, Technion, 32000 Haifa, Israel

e-mail: meshulam@math.technion.ac.il 


$$
\|x f\|_{2}\|\xi \widehat{f}\|_{2} \geq \frac{1}{4 \pi} .
$$

This classical inequality and its numerous extensions (see e.g. [3]) have major applications in diverse areas ranging from mathematical physics and differential equations to signal recovery and number theory. Here, we are concerned with discrete versions of the uncertainty principle. Let $G$ be a finite abelian group and let $\mathbb{F}[G]$ be the group algebra of $G$ over the field $\mathbb{F}$. For an element $f \in \mathbb{F}[G]$ let $T_{f}: \mathbb{F}[G] \rightarrow \mathbb{F}[G]$ be given by $T_{f} g=f g$. Let $A$ be a subset of $G$. Here and throughout the paper, we assume that $A \neq \emptyset$. The uncertainty number of $A \subset G$ is defined by

$$
u_{\mathbb{F}}(A)=\min \left\{\operatorname{rank} T_{f}: \varnothing \neq \operatorname{supp}(f) \subset A\right\} .
$$

The motivation for this definition is as follows. Let $m$ be the exponent of $G$ and suppose $\mathbb{F}$ contains a primitive $m$-th root of unity. Let $\widehat{G}$ denote the group of $\mathbb{F}$ valued characters of $G$. Identifying $\mathbb{F}[G]$ with the space of $\mathbb{F}$-valued functions on $G$, the Fourier transform of a function $f \in \mathbb{F}[G]$ is the function $\widehat{f} \in \mathbb{F}[\widehat{G}]$ given by $\widehat{f}(\chi)=\sum_{x \in G} \chi\left(x^{-1}\right) f(x)$. The characters $\chi \in \widehat{G}$ are eigenfunctions of $T_{f}$ with eigenvalues $f(\chi)$, hence $\operatorname{rank} T_{f}=|\operatorname{supp}(\widehat{f})|$. Therefore, in the semisimple case

$$
u_{\mathbb{F}}(A)=\min \{|\operatorname{supp}(\widehat{f})|: \emptyset \neq \operatorname{supp}(f) \subset A\} .
$$

The discrete counterpart of (1) (see e.g. [1]) asserts that for any $\mathbb{F}$ and $A \subset G$

$$
u_{\mathbb{F}}(A) \geq \frac{|G|}{|A|} .
$$

In the semisimple case (2) is equivalent to

$$
|\operatorname{supp}(f)| \cdot|\operatorname{supp}(\widehat{f})| \geq|G|
$$

for all nonzero $f$ 's. While (2) is sharp when $A$ is a coset of $G$, it can often be improved for particular choices of $G, A$, and $\mathbb{F}$. One such result (see [9]) states that if $p$ is prime and $A$ is a nonempty subset of the cyclic group $C_{p}$ then, $u_{\mathbb{C}}(A)=p-|A|+1$. See [7] for an extension to general abelian groups.

For a finite abelian group $G$, let $\Delta_{G}$ denote the $(|G|-1)$-dimensional simplex with vertex set $G$ and let $\Delta_{G}^{(j)}$ be the $j$-dimensional skeleton of $\Delta_{G}$. Let $A \subset G$ and let $1 \leq k \leq|G|$. The Sum Complex $X_{A, k}$ was defined in [6] by

$$
X_{A, k}=\Delta_{G}^{(k-2)} \cup\left\{\sigma \subset G:|\sigma|=k, \prod_{x \in \sigma} x \in A\right\} .
$$

Here, we obtain the following topological characterization of uncertainty numbers of subsets of $C_{p}$. 
Theorem 1.1 Let $A \subset C_{p}=G$. If $\mathbb{F}$ is algebraically closed, then

$$
u_{\mathbb{F}}(A)=p-\max \left\{1 \leq k \leq p: \tilde{H}_{k-1}\left(X_{A, k} ; \mathbb{F}\right) \neq 0\right\}
$$

If $\tilde{H}_{k-1}\left(X_{A, k} ; \mathbb{F}\right)=0$ for all $1 \leq k \leq p$, then the right-hand side of (3) is defined as p.

Example: Let $p=7$ and $A=\left\{1, z, z^{3}\right\} \subset C_{7}=\langle z\rangle$. The sum complex $X_{A, 3}$ is depicted in Figure (1b) where vertex label $\alpha$ corresponds to the element $z^{\alpha}$. Note that $X_{A, 3}$ is obtained from a 7-point triangulation of the real projective plane $\mathbb{R P}^{2}$ (Figure 1a) by adding the faces $\left\{z^{2}, z^{3}, z^{5}\right\},\left\{1, z^{2}, z^{6}\right\}$, and $\left\{z, z^{2}, z^{4}\right\} . X_{A, 3}$ is clearly homotopy equivalent to $\mathbb{R P}^{2}$, hence $H_{2}\left(X_{A, 3} ; \mathbb{F}_{2}\right) \neq 0$. Theorem 1.1 then implies that $u_{\overline{\mathbb{F}_{2}}}(A) \leq 4$. Together with the easy fact that $u_{\mathbb{F}}(B) \geq p-\max B$ for any $\mathbb{F}$ and $B \subset C_{p}$ it follows that $u_{\overline{\mathbb{F}_{2}}}(A)=4$. It can be checked that in fact $u_{\mathbb{F}_{2}}(A)=4$.

Let $z$ be a fixed generator of $C_{p}$ and let $A=\left\{a_{1}, \ldots, a_{m}\right\} \subset C_{p}$ where $a_{i}=z^{\alpha_{i}}$ and $\alpha_{i}$ is an element of the prime field $\mathbb{F}_{p}=\{0, \ldots, p-1\}$. Let $\alpha=\left(\alpha_{1}, \ldots, \alpha_{m}\right) \in \mathbb{F}_{p}^{m}$. The main ingredient in the proof of Theorem 1.1 is the computation of the homology of $X_{A, k}$ with arbitrary field coefficients. Let $\mathbb{F}$ be a field of characteristic $\ell$. First suppose that $\ell \neq p$ and let $\omega$ be a primitive $p$-th root of unity in the algebraic closure $\overline{\mathbb{F}}$. For $\beta=\left(\beta_{1}, \ldots, \beta_{k}\right) \in \mathbb{F}_{p}^{k}$ let $M_{\beta, \alpha}$ be the $k \times m$ matrix given by $M_{\beta, \alpha}(i, j)=\omega^{\beta_{i} \alpha_{j}}$. Let

$$
\mathscr{B}_{k}=\left\{\left(\beta_{1}, \ldots, \beta_{k}\right): 0 \leq \beta_{1}<\cdots<\beta_{k} \leq p-1\right\}
$$

The case $m=k$ of the following result is implicit in [6].

(a)

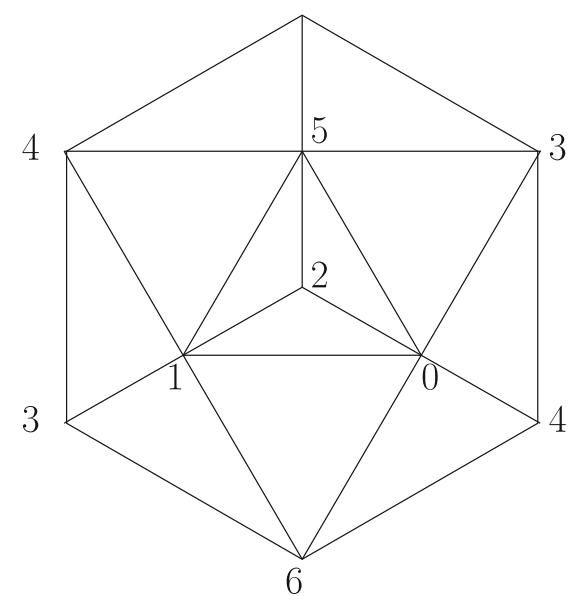

(b)

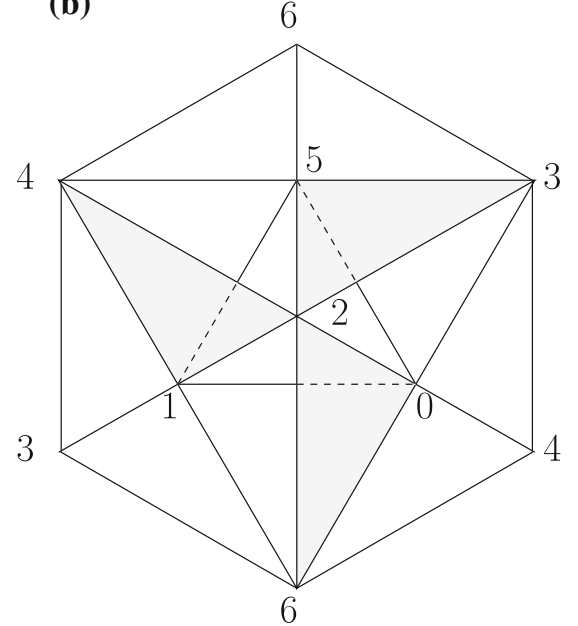

Fig. 1 a A 7-point triangulation of $\mathbb{R P}^{2}$. b $X_{A, 3}$ for $A=\left\{1, z, z^{3}\right\} \subset C_{7}$ 
Theorem 1.2 Let $A=\left\{z^{\alpha_{1}}, \ldots, z^{\alpha_{m}}\right\} \subset C_{p}$ and let $\alpha=\left(\alpha_{1}, \ldots, \alpha_{m}\right)$. If char $\mathbb{F} \neq p$ then for $1 \leq k \leq p$

$$
\operatorname{dim} \tilde{H}_{k-1}\left(X_{A, k} ; \mathbb{F}\right)=\frac{m}{k}\left(\begin{array}{l}
p-1 \\
k-1
\end{array}\right)-\frac{1}{p} \sum_{\beta \in \mathscr{B}_{k}} \operatorname{rank} M_{\beta, \alpha}
$$

Our main result concerns the homology of $X_{A, k}$ with $\mathbb{F}_{p}$ coefficients.

Theorem 1.3 Let $A \subset C_{p}$ such that $|A|=m$. Then for $1 \leq k \leq p$

$$
\operatorname{dim} \tilde{H}_{k-1}\left(X_{A, k} ; \mathbb{F}_{p}\right)=\left\{\begin{array}{cl}
0 & \text { if } m \leq k \\
\left(\frac{m}{k}-1\right)\left(\begin{array}{l}
p-1 \\
k-1
\end{array}\right) & \text { if } m>k
\end{array}\right.
$$

Remarks (1) The case $k=p$ of both Theorems 1.2 and 1.3 is straightforward. On one hand, since $X_{A, p}$ is a subcomplex of the $(p-1)$-simplex it follows that $\tilde{H}_{p-1}\left(X_{A, p} ; \mathbb{F}\right)=0$ for any $A$ and $\mathbb{F}$. On the other hand, let $\beta=(0,1, \ldots, p-1)$ be the unique element of $\mathscr{B}_{p}$. Then, $M_{\beta, \alpha}$ consists of $m$ full columns of the Fourier matrix, and thus has full rank $m$. Hence the right-hand side of (4) is also zero. In the sequel we will, therefore, assume that $1 \leq k<p$.

(2) The argument given in [6] for the case $m=k$ of Theorem 1.2 does not extend to the modular case. The approach here is different and is also utilized in the proof of our main result Theorem 1.3.

(3) The $f$-vector of $X_{A, k}$ satisfies $f_{i}\left(X_{A, k}\right)=\left(\begin{array}{c}p \\ i+1\end{array}\right)$ for $0 \leq i \leq k-2$ and $f_{k-1}\left(X_{A, k}\right)=\frac{m}{p}\left(\begin{array}{l}p \\ k\end{array}\right)=\frac{m}{k}\left(\begin{array}{c}p-1 \\ k-1\end{array}\right)$. The reduced Euler characteristic of $X_{A, k}$ is, therefore,

$$
\begin{aligned}
\tilde{\chi}\left(X_{A, k}\right) & =-1+\sum_{i=0}^{k-2}(-1)^{i}\left(\begin{array}{c}
p \\
i+1
\end{array}\right)+(-1)^{k-1} \frac{m}{p}\left(\begin{array}{l}
p \\
k
\end{array}\right) \\
& =(-1)^{k-1}\left(\frac{m}{k}-1\right)\left(\begin{array}{l}
p-1 \\
k-1
\end{array}\right) .
\end{aligned}
$$

Since $\tilde{H}_{i}\left(X_{A, k} ; \mathbb{F}_{p}\right)=0$ for $0 \leq i<k-2$ it follows that

$$
\begin{aligned}
\operatorname{dim} \tilde{H}_{k-2}\left(X_{A, k} ; \mathbb{F}_{p}\right) & =\operatorname{dim} \tilde{H}_{k-1}\left(X_{A, k} ; \mathbb{F}_{p}\right)-\left(\frac{m}{k}-1\right)\left(\begin{array}{l}
p-1 \\
k-1
\end{array}\right) N \\
& =\left\{\begin{array}{cl}
\left(1-\frac{m}{k}\right)\left(\begin{array}{l}
p-1 \\
k-1
\end{array}\right) & \text { if } m \leq k \\
0 & \text { if } m>k
\end{array}\right.
\end{aligned}
$$

(4) A classical result of Chebotarëv (see e.g. [8]) asserts that for $\mathbb{F}=\mathbb{Q}$ all $M_{\beta, \alpha}$ 's have full rank. Theorem 1.2, therefore, implies that (5) and (7) remain true for $\tilde{H}_{*}\left(X_{A, k} ; \mathbb{Q}\right)$.

(5) Theorem 1.3 does not hold for all fields $\mathbb{F}$ and in fact $\tilde{H}_{k-1}\left(X_{A, k} ; \mathbb{F}\right)$ may be nontrivial even if $k>|A|=m$. For example, using Theorem 1.2 it can be shown that if $A=\left\{1, z^{3}, z^{4}, z^{5}, z^{8}\right\} \subset C_{17}$ then $\tilde{H}_{7}\left(X_{A, 8} ; \mathbb{F}_{2}\right) \neq 0$. 
The paper is organized as follows. In Sect. 2 we consider $X_{A, k}$ where $A$ is a subset of an arbitrary abelian group $G$ and identify $\tilde{H}_{k-1}\left(X_{A, k} ; \mathbb{F}\right)$ with a certain subspace $\mathscr{H}(A)$ of skew-symmetric elements of the group algebra $\mathbb{F}\left[G^{k}\right]$. In Sect. 3 we use this characterization in the special case $G=C_{p}$ to prove Theorem 1.2. The proof of Theorem 1.3 given in Sect. 4 is more involved and depends additionally on some properties of generalized Vandermonde determinants over the group algebra $\mathbb{F}_{p}\left[C_{p}^{k}\right]$. Theorem 1.1 is derived in Sect. 5 as a direct consequence of Theorems 1.2 and 1.3. We conclude in Sect. 6 with some comments and open problems.

\section{A characterization of cycles}

Let $A$ be a nonempty subset of a finite abelian group $G$ and let $\mathbb{F}$ be a field. In this section, we provide a characterization (Claim 2.1) of the homology group $\tilde{H}_{k-1}\left(X_{A, k} ; \mathbb{F}\right)$ in terms of the group algebra $F\left[G^{k}\right]$. A simplified version valid under the assumption $\operatorname{gcd}(|G|, k)=1$ is given in Claim 2.2.

We first introduce some terminology. Fix $1 \leq k \leq|G|$. For an element $g \in G$ and $1 \leq i \leq k$ let $e_{i}(g)=(1, \ldots, 1, g, 1, \ldots, 1) \in G^{k}$ with $g$ appearing in the $i$-th coordinate. An element

$$
s=\sum_{\left(g_{1}, \ldots, g_{k}\right) \in G^{k}} s\left(g_{1}, \ldots, g_{k}\right)\left(g_{1}, \ldots, g_{k}\right) \in \mathbb{F}\left[G^{k}\right]
$$

is skew-symmetric if $s\left(g_{\sigma^{-1}(1)}, \ldots, g_{\sigma^{-1}(k)}\right)=\operatorname{sgn}(\sigma) s\left(g_{1}, \ldots, g_{k}\right)$ for all $\left(g_{1}, \ldots, g_{k}\right) \in G^{k}$ and $\sigma$ in the symmetric group $S_{k}$. If char $\mathbb{F}=2$ then $s$ is additionally required to satisfy $s\left(g_{1}, \ldots, g_{k}\right)=0$ if $g_{i}=g_{j}$ for some $i \neq j$. Let $\mathscr{S}$ denote the space of skew-symmetric elements of $\mathbb{F}\left[G^{k}\right]$. For $h \in G$ let $W_{h}=\left\{\left(g_{1}, \ldots, g_{k}\right) \in\right.$ $\left.G^{k}: \prod_{i=1}^{k} g_{i}=h\right\}$ and let $\mathscr{S}_{h}=\left\{s \in \mathscr{S}: \operatorname{supp}(s) \subset W_{h}\right\}$. Let $\rho_{h}$ denote the projection from $\mathscr{S}$ onto $\mathscr{S}_{h}$ given by $\rho_{h}\left(\sum_{u \in G^{k}} s(u) u\right)=\sum_{u \in W_{h}} s(u) u$.

Let $Y$ be a $(k-1)$-dimensional simplicial complex on the vertex set $V$ and let $Y(\ell)$ denote the set of its (unordered) $\ell$-simplices. Let $C_{k-1}(Y ; \mathbb{F})$ denote the space of $\mathbb{F}$ valued $(k-1)$-chains of $Y$. Recall that $\phi \in C_{k-1}(Y ; \mathbb{F})$ is a skew-symmetric $\mathbb{F}$-valued function on the ordered $(k-1)$-simplices of $Y$. A $(k-1)$-chain $\phi \in C_{k-1}(Y ; \mathbb{F})$ is a reduced $(k-1)$-cycle if for all $\left\{v_{1}, \ldots, v_{k-1}\right\} \in Y(k-2)$ it holds that

$$
\sum_{\left\{v_{k} \in V:\left\{v_{1}, \ldots, v_{k-1}, v_{k}\right\} \in Y(k-1)\right\}} \phi\left(v_{1}, \ldots, v_{k-1}, v_{k}\right)=0 .
$$

Specializing to the case $Y=X_{A, k}$ it is clear that

$$
C_{k-1}\left(X_{A, k}, \mathbb{F}\right)=\left\{s \in \mathscr{S}: \operatorname{supp}(s) \subset \cup_{a \in A} W_{a}\right\}=\bigoplus_{a \in A} \mathscr{S}_{a}
$$

By (8), $s \in C_{k-1}\left(X_{A, k}, \mathbb{F}\right)$ is a reduced $(k-1)$-cycle of $X_{A, k}$ if for all $\left(g_{1}, \ldots, g_{k-1}\right) \in$ $G^{k-1}$ 


$$
\sum_{a \in A} s\left(g_{1}, \ldots, g_{k-1}, a \prod_{j=1}^{k-1} g_{j}^{-1}\right)=0 .
$$

Let

$$
\mathscr{H}(A)=\left\{s \in \bigoplus_{a \in A} \mathscr{S}_{a}: \sum_{a \in A} e_{i}\left(a^{-1}\right) \rho_{a}(s)=0 \text { for all } 1 \leq i \leq k\right\} .
$$

The homology space $\tilde{H}_{k-1}\left(X_{A, k} ; \mathbb{F}\right)$ is characterized by the following

claim 2.1

$$
\tilde{H}_{k-1}\left(X_{A, k} ; \mathbb{F}\right)=\mathscr{H}(A)
$$

Proof First note that by skew symmetry

$$
\mathscr{H}(A)=\left\{s \in \bigoplus_{a \in A} \mathscr{S}_{a}: \sum_{a \in A} e_{k}\left(a^{-1}\right) \rho_{a}(s)=0\right\}
$$

Now let $s \in C_{k-1}\left(X_{A, k} ; \mathbb{F}\right)=\bigoplus_{a \in A} \mathscr{S}_{a}$. Then

$$
\begin{aligned}
& \sum_{a \in A} e_{k}\left(a^{-1}\right) \rho_{a}(s) \\
& =\sum_{a \in A} e_{k}\left(a^{-1}\right) \sum_{\left(g_{1}, \ldots, g_{k-1}\right) \in G^{k-1}} s\left(g_{1}, \ldots, g_{k-1}, a \prod_{j=1}^{k-1} g_{j}^{-1}\right)\left(g_{1}, \ldots, g_{k-1}, a \prod_{j=1}^{k-1} g_{j}^{-1}\right) \\
& =\sum_{\left(g_{1}, \ldots, g_{k-1}\right) \in G^{k-1}}\left(\sum_{a \in A} s\left(g_{1}, \ldots, g_{k-1}, a \prod_{j=1}^{k-1} g_{j}^{-1}\right)\right)\left(g_{1}, \ldots, g_{k-1}, \prod_{j=1}^{k-1} g_{j}^{-1}\right) .
\end{aligned}
$$

Therefore $\sum_{a \in A} e_{k}\left(a^{-1}\right) \rho_{a}(s)=0$ iff

$$
\sum_{a \in A} s\left(g_{1}, \ldots, g_{k-1}, a \prod_{j=1}^{k-1} g_{j}^{-1}\right)=0
$$

for all $\left(g_{1}, \ldots, g_{k-1}\right) \in G^{k-1}$. Hence the Claim follows from (9) and (10).

$$
\begin{aligned}
& \text { Let } \mathscr{S}^{A}=\bigoplus_{a \in A} \mathscr{S}=\left\{\left(r_{a}\right)_{a \in A}: r_{a} \in \mathscr{S}\right\} \text { and let } \\
& \qquad \mathscr{R}(A)=\left\{\left(r_{a}\right)_{a \in A} \in \mathscr{S}^{A}: \sum_{a \in A} e_{i}\left(a^{-1}\right) r_{a}=0 \text { for all } 1 \leq i \leq k\right\} .
\end{aligned}
$$


For $g \in G$ let $e(g)=\prod_{i=1}^{k} e_{i}(g)=(g, \ldots, g) \in G^{k}$. In Section 3 we shall need the following

claim 2.2 Assume that $\operatorname{gcd}(|G|, k)=1$. Then, the mapping $\Psi: \mathbb{F}[G] \otimes_{\mathbb{F}} \mathscr{H}(A) \rightarrow$ $\mathscr{R}(A)$ given by

$$
\Psi(g \otimes s)=\left(e(g) \rho_{a}(s)\right)_{a \in A}
$$

is an isomorphism.

Proof We first show injectivity. Let $w \in \operatorname{ker} \Psi$ and write $w=\sum_{g \in G} g \otimes s_{g}$ where $s_{g} \in \mathscr{H}(A)$. Then for all $a \in A$

$$
\sum_{g \in G} e(g) \rho_{a}\left(s_{g}\right)=0 .
$$

Since $e(g) \rho_{a}\left(s_{g}\right) \in \mathscr{S}_{a g^{k}}$ and $a g^{k} \neq a h^{k}$ for $g \neq h$ (by the assumption $\operatorname{gcd}(|G|, k)=$ $1)$, it follows that $e(g) \rho_{a}\left(s_{g}\right)=0$ and hence $\rho_{a}\left(s_{g}\right)=0$ for all $g \in G$ and $a \in A$. Therefore $w=0$. To show surjectivity let $\left(r_{a}\right)_{a \in A} \in \mathscr{R}(A)$. For $g \in G$ let

$$
s_{g}=e\left(g^{-1}\right) \sum_{a \in A} \rho_{a g^{k}}\left(r_{a}\right) \in \bigoplus_{a \in A} \mathscr{S}_{a}
$$

We first show that $s_{g} \in \mathscr{H}(A)$. For $1 \leq i \leq k$ and $g \in G$ let

$$
t_{i, g}=\sum_{a \in A} e_{i}\left(a^{-1}\right) \rho_{a g^{k}}\left(r_{a}\right) \in \mathscr{S}_{g^{k}} .
$$

Then

$$
\begin{aligned}
\sum_{g \in G} t_{i, g} & =\sum_{g \in G} \sum_{a \in A} e_{i}\left(a^{-1}\right) \rho_{a g^{k}}\left(r_{a}\right) \\
& =\sum_{a \in A} e_{i}\left(a^{-1}\right)\left(\sum_{g \in G} \rho_{a g^{k}}\left(r_{a}\right)\right)=\sum_{a \in A} e_{i}\left(a^{-1}\right) r_{a}=0 .
\end{aligned}
$$

It follows that $t_{i, g}=0$ for all $1 \leq i \leq k$ and $g \in G$. Therefore, for $1 \leq i \leq k$ and $g \in G$

$$
\begin{aligned}
\sum_{a \in A} e_{i}\left(a^{-1}\right) \rho_{a}\left(s_{g}\right) & =\sum_{a \in A} e_{i}\left(a^{-1}\right) \rho_{a}\left(e\left(g^{-1}\right) \sum_{a^{\prime} \in A} \rho_{a^{\prime} g^{k}}\left(r_{a^{\prime}}\right)\right) \\
& =\sum_{a \in A} e_{i}\left(a^{-1}\right) e\left(g^{-1}\right) \rho_{a g^{k}}\left(r_{a}\right)=e\left(g^{-1}\right) t_{i, g}=0 .
\end{aligned}
$$


Hence $s_{g} \in \mathscr{H}(A)$ and thus $w=\sum_{g \in G} g \otimes s_{g} \in \mathbb{F}[G] \bigotimes_{\mathbb{F}} \mathscr{H}(A)$. Finally, for all $a \in A$

$$
\sum_{g \in G} e(g) \rho_{a}\left(s_{g}\right)=\sum_{g \in G} e(g)\left(e\left(g^{-1}\right) \rho_{a g^{k}}\left(r_{a}\right)\right)=\sum_{g \in G} \rho_{a g^{k}}\left(r_{a}\right)=r_{a}
$$

and therefore, $\Psi(w)=\left(r_{a}\right)_{a \in A}$.

Corollary 2.3 If $\operatorname{gcd}(|G|, k)=1$ then

$$
\operatorname{dim} \tilde{H}_{k-1}\left(X_{A, k} ; \mathbb{F}\right)=\frac{\operatorname{dim} \mathscr{R}(A)}{|G|} .
$$

\section{The semisimple case}

Let $G$ be the cyclic group of prime order $C_{p}=\langle z\rangle$ and let $A=\left\{a_{1}, \ldots, a_{m}\right\} \subset C_{p}$ where $a_{j}=z^{\alpha_{j}}$. Let $\alpha=\left(\alpha_{1}, \ldots, \alpha_{k}\right) \in \mathbb{F}_{p}^{k}$. In this section, we compute $\operatorname{dim} \tilde{H}_{k-1}\left(X_{A, k} ; \mathbb{F}\right)$ when char $\mathbb{F} \neq p$. We may assume that $\mathbb{F}$ is algebraically closed. Recall that $\omega$ is a primitive $p$-th root of unity in $\mathbb{F}=\overline{\mathbb{F}}$. The character group $\widehat{C_{p}}$ consists of all characters $\eta_{u}$ where $u \in \mathbb{F}_{p}$ and $\eta_{u}(z)=\omega^{u}$. Similarly, $\widehat{C_{p}^{k}}=\left\{\chi_{\beta}: \beta=\left(\beta_{1}, \ldots, \beta_{k}\right) \in \mathbb{F}_{p}^{k}\right\}$ where for $\gamma=\left(\gamma_{1}, \ldots, \gamma_{k}\right) \in \mathbb{F}_{p}^{k}$

$$
\chi_{\beta}\left(z^{\gamma_{1}}, \ldots, z^{\gamma_{k}}\right)=\left(\eta_{\beta_{1}} \times \ldots \times \eta_{\beta_{k}}\right)\left(z^{\gamma_{1}}, \ldots, z^{\gamma_{k}}\right)=\omega^{\beta \gamma}
$$

and $\beta \gamma=\sum_{i=1}^{k} \beta_{i} \gamma_{i}$ is the standard inner product in $\mathbb{F}_{p}^{k}$. The Fourier transform of $f \in \mathbb{F}\left[C_{p}^{k}\right]$ is thus, $\widehat{f} \in \mathbb{F}\left[\widehat{C_{p}^{k}}\right]$ given by

$$
\widehat{f}\left(\chi_{\beta}\right)=\sum_{\gamma=\left(\gamma_{1}, \ldots, \gamma_{k}\right) \in \mathbb{F}_{p}^{k}} f\left(z^{\gamma_{1}}, \ldots, z^{\gamma_{k}}\right) \omega^{-\beta \gamma}
$$

As already remarked, in proving Theorem 1.2 we may assume that $k<p$. Corollary 2.3 then implies that $\operatorname{dim} \tilde{H}_{k-1}\left(X_{A, k} ; \mathbb{F}\right)=\frac{\operatorname{dim} \mathscr{R}(A)}{p}$. Theorem 1.2 will thus follow from

\section{Proposition 3.1}

$$
\operatorname{dim} \mathscr{R}(A)=m\left(\begin{array}{l}
p \\
k
\end{array}\right)-\sum_{\beta \in \mathscr{B}_{k}} \operatorname{rank} M_{\beta, \alpha}
$$

Proof Define an $\mathbb{F}$-linear mapping

$$
\Phi: \mathscr{S}^{A} \rightarrow \bigoplus_{\beta \in \mathscr{B}_{k}} \mathbb{F}^{m}
$$


as follows. For $r=\left(r_{a_{j}}\right)_{j=1}^{m} \in \mathscr{S}^{A}$ let

$$
\Phi(r)=\left(\left[\begin{array}{c}
\widehat{r_{a_{1}}}\left(\chi_{\beta}\right) \\
\vdots \\
\widehat{r_{a_{m}}}\left(\chi_{\beta}\right)
\end{array}\right]: \beta \in \mathscr{B}_{k}\right) \text {. }
$$

Note that since $r_{a_{j}}$ is a skew-symmetric element of $\mathbb{F}\left[C_{p}^{k}\right]$, it follows that $\widehat{r_{a_{j}}}$ is a skew symmetric element of $\mathbb{F}\left[\widehat{C_{p}^{k}}\right]$ and hence is determined by its values on $\mathscr{B}_{k}$. Therefore, $\Phi$ is an isomorphism.

claim 3.2 $\Phi$ restricts to an isomorphism from $\mathscr{R}(A)$ onto $\bigoplus_{\beta \in \mathscr{B}_{k}} \operatorname{ker} M_{\beta, \alpha}$.

Proof Note that if $1 \leq i \leq k$ and $\beta=\left(\beta_{1}, \ldots, \beta_{k}\right) \in \mathbb{F}_{p}^{k}$ then

$$
\widehat{e_{i}\left(a_{j}^{-1}\right)}\left(\chi_{\beta}\right)=\widehat{e_{i}\left(z^{-\alpha_{j}}\right)}\left(\chi_{\beta}\right)=\omega^{\beta_{i} \alpha_{j}}
$$

Let $r=\left(r_{a_{j}}\right)_{j=1}^{m} \in \mathscr{S}^{A}$ and fix $1 \leq i \leq k$ and $\beta=\left(\beta_{1}, \ldots, \beta_{k}\right) \in \mathbb{F}_{p}^{k}$. Evaluating the Fourier transform of the element $\sum_{j=1}^{m} e_{i}\left(a_{j}^{-1}\right) r_{a_{j}}$ at the character $\chi_{\beta}$ we obtain

$$
\begin{aligned}
& \left(\sum_{j=1}^{m} e_{i}\left(a_{j}^{-1}\right) r_{a_{j}}\right) \wedge\left(\chi_{\beta}\right) \\
& =\sum_{j=1}^{m} \widehat{e_{i}\left(a_{j}^{-1}\right)}\left(\chi_{\beta}\right) \widehat{r_{a_{j}}}\left(\chi_{\beta}\right) \\
& =\sum_{j=1}^{m} \omega^{\beta_{i} \alpha_{j} \widehat{r_{a_{j}}}\left(\chi_{\beta}\right) .} .
\end{aligned}
$$

It follows that $r=\left(r_{a_{j}}\right)_{j=1}^{m} \in \mathscr{R}(A)$ iff for all $\beta=\left(\beta_{1}, \ldots, \beta_{k}\right) \in \mathbb{F}_{p}^{k}$

$$
M_{\beta, \alpha}\left[\begin{array}{c}
\widehat{r_{a_{1}}}\left(\chi_{\beta}\right) \\
\vdots \\
\widehat{r_{a_{m}}}\left(\chi_{\beta}\right)
\end{array}\right]=\left[\begin{array}{ccc}
\omega^{\beta_{1} \alpha_{1}} & \cdots & \omega^{\beta_{1} \alpha_{m}} \\
\vdots & \ddots & \vdots \\
\omega^{\beta_{k} \alpha_{1}} & \cdots & \omega^{\beta_{k} \alpha_{m}}
\end{array}\right]\left[\begin{array}{c}
\widehat{r_{a_{1}}}\left(\chi_{\beta}\right) \\
\vdots \\
\widehat{r_{a_{m}}}\left(\chi_{\beta}\right)
\end{array}\right]=0
$$

Therefore, $r=\left(r_{a_{j}}\right)_{j=1}^{m} \in \mathscr{R}(A)$ iff $\Phi(r) \in \bigoplus_{\beta \in \mathscr{B}_{k}}$ ker $M_{\beta, \alpha}$. The Claim now follows from the bijectivity of $\Phi$.

Proof of Proposition 3.1: By Claim 3.2

$$
\operatorname{dim} \mathscr{R}(A)=\sum_{\beta \in \mathscr{B}_{k}} \operatorname{dim} \operatorname{ker} M_{\beta, \alpha}=\sum_{\beta \in \mathscr{B}_{k}}\left(m-\operatorname{rank} M_{\beta, \alpha}\right)
$$




$$
=m\left(\begin{array}{l}
p \\
k
\end{array}\right)-\sum_{\beta \in \mathscr{B}_{k}} \operatorname{rank} M_{\beta, \alpha} .
$$

\section{The modular case}

In subsections 4.1 and 4.2 we study certain properties of determinants of generalized Vandermonde matrices over the group algebra $\mathbb{F}_{p}\left[C_{p}^{k}\right]$. These results are then used in subsection 4.3 to prove Theorem 1.3.

\subsection{A generalized Vandermonde}

Recall that $z$ is a fixed generator of $C_{p}$ and let $1 \leq k \leq p$. For $1 \leq i \leq k$ let $x_{i}=e_{i}(z)$. Then $\left\{x_{1}, \ldots, x_{k}\right\}$ is a generating set of $C_{p}^{k}$. Let $x=\left(x_{1}, \ldots, x_{k}\right)$. For $\beta=\left(\beta_{1}, \ldots, \beta_{k}\right) \in \mathscr{B}_{k}$ let

$$
N_{\beta}=\left[\begin{array}{ccc}
x_{1}^{-\beta_{1}} & \cdots & x_{1}^{-\beta_{k}} \\
\vdots & \ddots & \vdots \\
x_{k}^{-\beta_{1}} & \cdots & x_{k}^{-\beta_{k}}
\end{array}\right]
$$

Proposition 4.1 Let $1 \leq k \leq p$. Then

$$
\operatorname{det} N_{\beta}=w_{\beta} \prod_{1 \leq i<j \leq k}\left(x_{i}-x_{j}\right)
$$

where $w_{\beta}$ is a unit of $\mathbb{F}_{p}\left[C_{p}^{k}\right]$.

Recall the definition of Schur polynomials (see e.g., [2]). Let $\xi=\left(\xi_{1}, \ldots, \xi_{k}\right)$ be a vector of variables. For a partition $\lambda=\left(\lambda_{1} \geq \cdots \geq \lambda_{k}\right)$ let

$$
D_{\lambda}(\xi)=D_{\lambda}\left(\xi_{1}, \ldots, \xi_{k}\right)=\operatorname{det}\left(\left[\xi_{i}^{\lambda_{j}+k-j}\right]_{i, j=1}^{k}\right) \in \mathbb{Z}\left[\xi_{1}, \ldots, \xi_{k}\right]
$$

Note that for the zero partition $0=(0, \ldots, 0)$

$$
D_{0}(\xi)=\operatorname{det}\left[\begin{array}{cccc}
\xi_{1}^{k-1} & \xi_{1}^{k-2} & \cdots & 1 \\
\vdots & \vdots & \ddots & \vdots \\
\xi_{k}^{k-1} & \xi_{k}^{k-2} & \cdots & 1
\end{array}\right]=\prod_{1 \leq i<j \leq k}\left(\xi_{i}-\xi_{j}\right)
$$

The Schur polynomial associated with $\lambda$ is

$$
\mathbb{S}_{\lambda}(\xi)=\frac{D_{\lambda}(\xi)}{D_{0}(\xi)} \in \mathbb{Z}\left[\xi_{1}, \ldots, \xi_{k}\right]
$$


The dimension formula (see e.g., Proposition 5.21.2 in [2]) asserts that

$$
\mathbb{S}_{\lambda}(1, \ldots, 1)=\prod_{1 \leq i<j \leq k} \frac{\lambda_{i}-\lambda_{j}+j-i}{j-i}
$$

Proof of Proposition 4.1: Let

$$
\lambda=\left(\lambda_{1}, \ldots, \lambda_{k}\right)=\left(p-\beta_{1}-k+1, p-\beta_{2}-k+2, \ldots, p-\beta_{k}\right) .
$$

Note that $\beta=\left(\beta_{1}, \cdots, \beta_{k}\right) \in \mathscr{B}_{k}$ implies that $\lambda_{1} \geq \cdots \geq \lambda_{k}$. Then

$$
\begin{aligned}
\operatorname{det} N_{\beta} & =D_{\lambda}(x)=\mathbb{S}_{\lambda}(x) D_{0}(x) \\
& =\mathbb{S}_{\lambda}(x) \prod_{1 \leq i<j \leq k}\left(x_{i}-x_{j}\right) .
\end{aligned}
$$

By (13) the image of $\mathbb{S}_{\lambda}(x) \in \mathbb{F}_{p}\left[C_{p}^{k}\right]$ under the augmentation map $\mathbb{F}_{p}\left[C_{p}^{k}\right] \rightarrow \mathbb{F}_{p}$ is

$$
\mathbb{S}_{\lambda}(1, \ldots, 1)(\bmod p)=\prod_{1 \leq i<j \leq k} \frac{\beta_{j}-\beta_{i}}{j-i}(\bmod p) \neq 0(\bmod p)
$$

It follows that $w_{\beta}=\mathbb{S}_{\lambda}(x)$ is invertible in $\mathbb{F}_{p}\left[C_{p}^{k}\right]$.

\subsection{Skew-symmetric annihilators of $\mathbf{D}_{\mathbf{0}}(\mathbf{x})$}

Recall that $\mathscr{S}$ is the space of skew-symmetric elements of $\mathbb{F}_{p}\left[C_{p}^{k}\right]$. In this subsection we show

Proposition 4.2 Assume that $1 \leq k<p$ and let $s \in \mathscr{S}$. If $D_{0}(x) s=0$ then $s=0$.

The proof of Proposition 4.2 depends on Proposition 4.3 below. Let $\mathbb{N}$ denote the nonnegative integers and for $a, b \in \mathbb{N}$ let $[a, b]=\{a, \ldots, b\}$. Let

$$
\mathbb{N}_{k}=\left\{\left(\mu_{1}, \ldots, \mu_{k}\right) \in \mathbb{N}^{k}: \mu_{i} \neq \mu_{j} \text { if } i \neq j\right\}
$$

For $\mu=\left(\mu_{1}, \ldots, \mu_{k}\right), v=\left(v_{1}, \ldots, v_{k}\right) \in \mathbb{N}_{k}$ write $\mu \preceq v$ if $\left\{\mu_{1}, \ldots, \mu_{k}\right\}$ precedes $\left\{v_{1}, \ldots, v_{k}\right\}$ in the lexicographic order on $k$-subsets of $\mathbb{N}$, i.e., if

$$
\sum_{i=1}^{k} 2^{-\mu_{i}} \geq \sum_{i=1}^{k} 2^{-v_{i}}
$$

Fix a $\mu=\left(\mu_{1}, \ldots, \mu_{k}\right) \in \mathbb{N}_{k}$ such that $\mu_{1}<\cdots<\mu_{k}$ and let

$$
L=\left\{1 \leq i \leq k-1: \mu_{i}+1<\mu_{i+1}\right\}
$$


Write $L=\left\{\ell_{1}<\ldots<\ell_{t-1}\right\}$ and let $\ell_{0}=0, \ell_{t}=k$. For $1 \leq i \leq t$ let $K_{i}=\left[\ell_{i-1}+1, \ell_{i}\right]$. Let

$$
\mathscr{G}_{1}(\mu)=\left\{(\gamma, \sigma) \in \mathbb{N}_{k} \times S_{k}: \gamma \preceq \mu \text { and } \gamma_{j}-\sigma(j)=\mu_{j}-j \text { for all } j\right\} .
$$

We will need the following characterization of $\mathscr{G}_{1}(\mu)$. Let $S_{K}$ denote the symmetric group on a set $K$. Let $T=S_{K_{1}} \times \cdots \times S_{K_{t}}$ be the Young subgroup of $S_{k}$ corresponding to the partition $[k]=\cup_{i=1}^{t} K_{i}$. Let

$$
\mathscr{G}_{2}(\mu)=\left\{(\gamma, \sigma) \in \mathbb{N}_{k} \times T: \gamma_{j}=\mu_{\sigma(j)} \text { for all } j\right\} .
$$

Proposition 4.3 $\mathscr{G}_{1}(\mu)=\mathscr{G}_{2}(\mu)$

Proof We first show that $\mathscr{G}_{2}(\mu) \subset \mathscr{G}_{1}(\mu)$. Let $(\gamma, \sigma) \in \mathscr{G}_{2}(\mu)$ and let $1 \leq j \leq k$. If $j \in K_{i}$ then $\sigma(j) \in K_{i}$ and hence $\mu_{\sigma(j)}-\mu_{j}=\sigma(j)-j$. Therefore,

$$
\gamma_{j}-\sigma(j)=\mu_{\sigma(j)}-\sigma(j)=\mu_{j}-j
$$

and so $(\gamma, \sigma) \in \mathscr{G}_{1}(\mu)$. For the other direction let $(\gamma, \sigma) \in \mathscr{G}_{1}(\mu)$. Write $\gamma=$ $\left(\gamma_{1}, \ldots, \gamma_{k}\right)$ and let $\pi \in S_{k}$ such that $\gamma_{\pi(1)}<\cdots<\gamma_{\pi(k)}$.

claim 4.4 For $1 \leq i \leq t$ and $j \in K_{i}$

(a) $\sigma(\pi(j))=j$.

(b) $\gamma_{\pi(j)}=\mu_{j}$.

(c) $\pi(j) \in K_{i}$.

Proof We argue by induction on $j$. Suppose (a),(b), and (c) hold for all $j^{\prime}<j$. (a) implies that $\left\{\sigma\left(\pi\left(j^{\prime}\right)\right): j^{\prime}<j\right\}=[j-1]$ and hence $\sigma(\pi(j)) \geq j$. Therefore,

$$
\mu_{j}-j \geq \mu_{j}-\sigma(\pi(j))
$$

Next note that by (b) $\gamma_{\pi\left(j^{\prime}\right)}=\mu_{j^{\prime}}$ for all $j^{\prime}<j$. As $\gamma \preceq \mu$ it follows that $\mu_{j} \geq \gamma_{\pi(j)}$, and therefore,

$$
\mu_{j}-\sigma(\pi(j)) \geq \gamma_{\pi(j)}-\sigma(\pi(j))=\mu_{\pi(j)}-\pi(j) .
$$

Finally (c) implies that $\left\{\pi\left(j^{\prime}\right): 1 \leq j^{\prime} \leq \ell_{i-1}\right\}=\left[1, \ell_{i-1}\right]$ and therefore $\pi(j) \geq$ $\ell_{i-1}+1$. Together with the assumption $j \in K_{i}$ it follows that

$$
\mu_{\pi(j)}-\pi(j) \geq \mu_{\ell_{i-1}+1}-\left(\ell_{i-1}+1\right)=\mu_{j}-j .
$$

It follows that the three inequalities in (14),(15),(16) are in fact equalities. Therefore $\sigma(\pi(j))=j, \gamma_{\pi(j)}=\mu_{j}$ and $\mu_{\pi(j)}=\mu_{j}+(\pi(j)-j)$, respectively, establishing (a),(b), and (c) for $j$.

Claim 4.4 implies that $\sigma=\pi^{-1} \in T$ and that $\gamma_{j}=\mu_{\sigma(j)}$ for all $1 \leq j \leq k$. Therefore $(\gamma, \sigma) \in \mathscr{G}_{2}(\mu)$. 
Proof of Proposition 4.2: Let $s \in \mathscr{S}$ such that $D_{0}(x) s=0$. We have to show that $s=0$. For $\gamma=\left(\gamma_{1}, \ldots, \gamma_{k}\right) \in \mathbb{F}_{p}^{k}$ we abbreviate $x^{\gamma}=\prod_{j=1}^{k} x_{j}^{\gamma_{j}}=\left(z^{\gamma_{1}}, \ldots, z^{\gamma_{k}}\right) \in$ $C_{p}^{k}$. Note that this notation is unambiguous since $x_{j}^{p}=1$. By assumption

$$
\begin{aligned}
0=D_{0}(x) s & =\sum_{\sigma \in S_{k}} \operatorname{sgn}(\sigma) \prod_{j=1}^{k} x_{j}^{k-\sigma(j)} \sum_{\gamma=\left(\gamma_{1}, \ldots, \gamma_{k}\right) \in \mathbb{F}_{p}^{k}} s\left(x^{\gamma}\right) \prod_{j=1}^{k} x_{j}^{\gamma_{j}} \\
& =\sum_{\gamma=\left(\gamma_{1}, \ldots, \gamma_{k}\right) \in \mathbb{F}_{p}^{k}} \sum_{\sigma \in S_{k}} \operatorname{sgn}(\sigma) s\left(x^{\gamma}\right) \prod_{j=1}^{k} x_{j}^{\gamma_{j}+k-\sigma(j)} .
\end{aligned}
$$

Suppose for contradiction that $s \neq 0$ and let

$$
\mu=\left(\mu_{1}, \ldots, \mu_{k}\right)=\max \left\{\gamma \in \mathscr{B}_{k}: s\left(x^{\gamma}\right) \neq 0\right\},
$$

where the maximum is taken with respect to $\preceq$. Let $\lambda \in \mathbb{F}_{p}$ denote the coefficient of $\prod_{j=1}^{k} x_{j}^{\mu_{j}+k-j}$ in the expansion of $D_{0}(x) s$ in the standard basis $\left\{x^{\beta}: \beta \in \mathbb{F}_{p}^{k}\right\}$ of $\mathbb{F}_{p}\left[C_{p}^{k}\right]$. Note that if

$$
\prod_{j=1}^{k} x_{j}^{\mu_{j}+k-j}=\prod_{j=1}^{k} x_{j}^{\gamma_{j}+k-\sigma(j)}
$$

then for all $1 \leq j \leq k$

$$
\mu_{j}-j=\gamma_{j}-\sigma(j)(\bmod p)
$$

Since

$$
-1 \leq \mu_{j}-j \leq p-1-k
$$

and

$$
-k \leq \gamma_{j}-\sigma(j) \leq p-2
$$

it follows that

$$
\mu_{j}-j=\gamma_{j}-\sigma(j)
$$

Hence, Eq.(17) and Proposition 4.3 imply that

$$
\begin{aligned}
\lambda & =\sum_{(\gamma, \sigma) \in \mathscr{G}_{1}(\mu)} \operatorname{sgn}(\sigma) s\left(x^{\gamma}\right)=\sum_{(\gamma, \sigma) \in \mathscr{G}_{2}(\mu)} \operatorname{sgn}(\sigma) s\left(x^{\gamma}\right) \\
& =\sum_{(\gamma, \sigma) \in \mathscr{G}_{2}(\mu)} \operatorname{sgn}(\sigma) s\left(z^{\gamma_{1}}, \ldots, z^{\gamma_{k}}\right) \\
& =\sum_{\sigma \in S_{K_{1}} \times \cdots \times S_{K_{t}}} \operatorname{sgn}(\sigma) s\left(z^{\mu_{\sigma(1)}}, \ldots, z^{\mu_{\sigma(k)}}\right)
\end{aligned}
$$




$$
=\left|S_{K_{1}} \times \cdots \times S_{K_{t}}\right| s\left(z^{\mu_{1}}, \ldots, z^{\mu_{k}}\right)=\prod_{i=1}^{t}\left(\ell_{i}-\ell_{i-1}\right) ! s\left(x^{\mu}\right) .
$$

Since $\ell_{t}=k<p$ it follows that $\prod_{i=1}^{t}\left(\ell_{i}-\ell_{i-1}\right) ! \neq 0(\bmod p)$ and so $\lambda \neq 0$. Therefore, $D_{0}(x) s \neq 0$, a contradiction.

Remarks Proposition 4.2 does not hold for $k=p$. Indeed, in this case $s=D_{0}(x)=$ $\prod_{1 \leq i<j \leq p}\left(x_{i}-x_{j}\right)$ is a nonzero skew-symmetric element of $\mathbb{F}_{p}\left[C_{p}^{p}\right]$ and it can be checked that $D_{0}(x) s=D_{0}(x)^{2}=0$.

\subsection{Homology of $\mathbf{X}_{\mathbf{A}, \mathbf{k}}$ over $\mathbb{F}_{\mathbf{p}}$}

In this subsection, we prove Theorem 1.3. We first consider the case $m=k$.

Theorem 4.5 If $|A|=k$ then $\tilde{H}_{k-1}\left(X_{A, k} ; \mathbb{F}_{p}\right)=0$.

Proof As already noted the case $k=p$ is trivial so we assume $k<p$. Let $A=$ $\left\{a_{1}, \ldots, a_{k}\right\}$ where $a_{i}=z^{\alpha_{i}}$ and $\alpha=\left(\alpha_{1}, \ldots, \alpha_{k}\right) \in \mathscr{B}_{k}$. Let $s \in \tilde{H}_{k-1}\left(X_{A, k} ; \mathbb{F}_{p}\right)$ then by Claim 2.1

$$
\begin{aligned}
N_{\alpha}\left[\begin{array}{c}
\rho_{a_{1}}(s) \\
\vdots \\
\rho_{a_{k}}(s)
\end{array}\right] & =\left[\begin{array}{ccc}
x_{1}^{-\alpha_{1}} & \cdots & x_{1}^{-\alpha_{k}} \\
\vdots & \ddots & \vdots \\
x_{k}^{-\alpha_{1}} & \cdots & x_{k}^{-\alpha_{k}}
\end{array}\right]\left[\begin{array}{c}
\rho_{a_{1}}(s) \\
\vdots \\
\rho_{a_{k}}(s)
\end{array}\right] \\
& =\left[\begin{array}{ccc}
e_{1}\left(a_{1}^{-1}\right) & \cdots & e_{1}\left(a_{k}^{-1}\right) \\
\vdots & \ddots & \vdots \\
e_{k}\left(a_{1}^{-1}\right) & \cdots & e_{k}\left(a_{k}^{-1}\right)
\end{array}\right]\left[\begin{array}{c}
\rho_{a_{1}}(s) \\
\vdots \\
\rho_{a_{k}}(s)
\end{array}\right]=0 .
\end{aligned}
$$

Therefore, $\operatorname{det} N_{\alpha} \cdot \rho_{a_{j}}(s)=0$ for all $1 \leq j \leq k$. Proposition 4.1 then implies that $D_{0}(x) \rho_{a_{j}}(s)=0$. Hence $\rho_{a_{j}}(s)=0$ by Proposition 4.2. It follows that $s=0$ and so $\tilde{H}_{k-1}\left(X_{A, k} ; \mathbb{F}_{p}\right)=0$.

Proof of Theorem 1.3 Let $|A|=m \geq k$ and let $A^{\prime}$ be an arbitrary subset of $A$ of cardinality $k$. Theorem 4.5 and Eq. (6) imply that $\tilde{H}_{*}\left(X_{A^{\prime}, k} ; \mathbb{F}_{p}\right)=0$. Hence by the exact sequence

$$
\begin{aligned}
0= & \tilde{H}_{k-1}\left(X_{A^{\prime}, k} ; \mathbb{F}_{p}\right) \rightarrow \tilde{H}_{k-1}\left(X_{A, k} ; \mathbb{F}_{p}\right) \rightarrow \\
& \rightarrow \tilde{H}_{k-1}\left(X_{A, k}, X_{A^{\prime}, k} ; \mathbb{F}_{p}\right) \rightarrow \tilde{H}_{k-2}\left(X_{A^{\prime}, k} ; \mathbb{F}_{p}\right)=0
\end{aligned}
$$

it follows that

$$
\begin{aligned}
\operatorname{dim} \tilde{H}_{k-1}\left(X_{A, k} ; \mathbb{F}_{p}\right) & =\operatorname{dim} \tilde{H}_{k-1}\left(X_{A, k}, X_{A^{\prime}, k} ; \mathbb{F}_{p}\right) \\
& =f_{k-1}\left(X_{A, k}\right)-f_{k-1}\left(X_{A^{\prime}, k}\right)=\left(\frac{m}{k}-1\right)\left(\begin{array}{l}
p-1 \\
k-1
\end{array}\right) .
\end{aligned}
$$




\section{Uncertainty numbers and homology}

Proof of Theorem 1.1 Recall that $A=\left\{z^{\alpha_{1}}, \ldots, z^{\alpha_{m}}\right\} \subset C_{p}$. Let $\mathbb{F}$ be an algebraically closed field with char $\mathbb{F}=\ell$. We consider two cases:

(i) The semisimple case $\ell \neq p$. Here, it suffices to show that for any fixed $1 \leq$ $k \leq p$ the following three conditions are equivalent:

(C1) $\tilde{H}_{k-1}\left(X_{A, k} ; \mathbb{F}\right) \neq 0$.

(C2) There exists a $\beta \in \mathscr{B}_{k}$ such that rank $M_{\beta, \alpha}<m$.

(C3) $u_{\mathbb{F}}(A) \leq p-k$.

First note that Theorem 1.2 implies that $\tilde{H}_{k-1}\left(X_{A, k} ; \mathbb{F}\right) \neq 0$ iff

$$
\frac{m}{p}\left(\begin{array}{l}
p \\
k
\end{array}\right)>\frac{1}{p} \sum_{\beta \in \mathscr{B}_{k}} \operatorname{rank} M_{\beta, \alpha} .
$$

This proves the equivalence of (C1) and (C2). Next, let $\lambda=\left(\lambda_{1}, \ldots, \lambda_{m}\right) \in \mathbb{F}^{m}$ and let $f_{\lambda}=\sum_{j=1}^{m} \lambda_{j} z^{\alpha_{j}} \in \mathbb{F}\left[C_{p}\right]$. Then, $\operatorname{supp}\left(f_{\lambda}\right) \subset A$ and for $\beta=\left(\beta_{1}, \ldots, \beta_{k}\right) \in \mathbb{F}_{p}^{k}$

$$
M_{\beta, \alpha} \lambda=\left(\sum_{j=1}^{m} \lambda_{j} \omega^{\beta_{i} \alpha_{j}}\right)_{i=1}^{k}=\left(\widehat{f_{\lambda}}\left(\eta_{-\beta_{i}}\right)\right)_{i=1}^{k}
$$

It follows that if $\beta=\left(\beta_{1}, \ldots, \beta_{k}\right) \in \mathscr{B}_{k}$ then rank $M_{\beta, \alpha}<m$ iff there exists a nonzero $f=f_{\lambda} \in \mathbb{F}\left[C_{p}\right]$ such that $\operatorname{supp}(f) \subset A$ and $\operatorname{supp}(f) \cap\left\{\eta_{-} \beta_{i}\right\}_{i=1}^{k}=\emptyset$. This proves the equivalence of (C2) and (C3).

(ii) The modular case $\ell=p$. Let $\mathbb{F}$ be a field of characteristic $p$. By Theorem 1.3

$$
p-\max \left\{k: \tilde{H}_{k-1}\left(X_{A, k} ; \mathbb{F}\right) \neq 0\right\}=p-m+1
$$

It thus suffices to show that $u_{\mathbb{F}}(A)=p-m+1$. Let $0 \neq f=\sum_{j=1}^{m} \lambda_{j} z^{\alpha_{j}} \in \mathbb{F}\left[C_{p}\right]$. Regarding $f=f(z)$ as an element of the polynomial ring $\mathbb{F}[z]$, the rank of $T_{f}$ is given by

$$
\begin{aligned}
\operatorname{rank} T_{f} & =p-\operatorname{deg} \operatorname{gcd}\left(f(z), z^{p}-1\right) \\
& =p-\operatorname{deg} \operatorname{gcd}\left(f(z),(z-1)^{p}\right)=p-\mu(f),
\end{aligned}
$$

where $\mu(f)$ is the multiplicity of 1 as a root of $f(z)$. By a simple well-known result (see e.g., Lemma 2 in [4]), $\mu(f) \leq m-1$ and hence $u_{\mathbb{F}}(A) \geq p-m+1$. For the other direction note that the $\mathbb{F}$-linear space

$$
\mathscr{P}=\left\{f(z) \in \mathbb{F}\left[C_{p}\right]: \mu(f) \geq m-1\right\}
$$

satisfies $\operatorname{dim}_{\mathbb{F}} \mathscr{P}=p-m+1$ and hence must contain a nonzero $f$ of the form $f(z)=\sum_{j=1}^{m} \lambda_{j} z^{\alpha_{j}}$. It follows from (18) that $f$ satisfies rank $T_{f} \leq p-m+1$. Therefore, $u_{\mathbb{F}}(A) \leq p-m+1$. 


\section{Concluding remarks}

We mention two problems related to the results of this paper.

1. Let $k \geq 2$ and let $X$ be a $(k-1)$-dimensional complex $X$ with $N=f_{k-1}(X)$ facets. It was observed by G. Kalai, S. Weinberger and the author that the torsion subgroup $H_{k-2}(X)_{\text {tor }}$ satisfies $\left|H_{k-2}(X)_{\text {tor }}\right| \leq \sqrt{k}^{N}$. Kalai on the other hand showed [5] that there exist $X$ 's with $\left|H_{k-2}(X)_{t o r}\right| \geq \sqrt{k / e}^{N}$. Computer experiments indicate that the $\mathbb{Q}$-acyclic sum complexes obtained by taking $|A|=k$ often have large torsion. For example, $A=\left\{1, z, z^{19}\right\} \subset C_{83}$ satisfies $\left|H_{1}\left(X_{A, 3}\right)\right|>1.17^{N}$ where $N=f_{2}\left(X_{A, 3}\right)=\left(\begin{array}{c}82 \\ 2\end{array}\right)$. Note that the base of the exponent 1.17 is slightly bigger than the constant $\sqrt{3 / e} \doteq 1.05$ in Kalai's lower bound. In view of this, it would be interesting to determine (or estimate) the maximum torsion of sum complexes.

2. Theorem 1.1 characterizes the uncertainty number $u_{\mathbb{F}}(A)$ with $A \subset G=C_{p}$ and $\mathbb{F}$ algebraically closed, in terms of the homology of $X_{A, k}$ over $\mathbb{F}$. It would be useful to find appropriate extensions of this characterization to general finite groups $G$ and arbitrary fields $\mathbb{F}$.

Acknowledgments Research supported by a grant from the Israel Science Foundation with additional partial support from ERC Advanced Research Grant No. 267165 (DISCONV) and ESF grant (ACAT). The author would like to thank Shmuel Weinberger for helpful discussions.

\section{References}

1. Donoho, D.L., Stark, P.B.: Uncertainty principles and signal recovery. SIAM J. Appl. Math. 49, 906-931 (1989)

2. Etingof, P.: Introduction to Representation Theory, Student Mathematical Library, vol. 59. AMS, Salt Lake (2011)

3. Folland, G., Sitaram, A.: The uncertainty principle: a mathematical survey. J. Fourier Anal. Appl. 3, 207-238 (1997)

4. Frenkel, P.E.: Simple proof of Chebotarev's theorem on roots of unity, arXiv:math/0312398

5. Kalai, G.: Enumeration of $\mathbb{Q}$-acyclic simplicial complexes. Israel J. Math. 45, 337-351 (1983)

6. Linial, N., Meshulam, R., Rosenthal, M.: Sum complexes: a new family of hypertrees. Discrete Comput. Geom. 44, 622-636 (2010)

7. Meshulam, R.: An uncertainty inequality for finite Abelian groups. Eur. J. Comb. 27, 63-67 (2006)

8. Stevenhagen, P., Lenstra, H.W.: Chebotarëv and his density theorem. Math. Intell. 18, $26-37$ (1996)

9. Tao, T.: An uncertainty principle for cyclic groups of prime order. Math. Res. Lett. 12, 121-127 (2005) 